\title{
Study Protocol: Traditional Chinese Medicine (TCM) Syndrome Differentiation for Heart Failure patients and its Implication for Long-term Therapeutic Outcome of Qiliqiangxin Capsules
}

\author{
Alice Yeuk Lan Leung \\ The University of Hong Kong School of Chinese Medicine \\ Hoiyong Chen \\ The University of Hong Kong School of Chinese Medicine \\ Zhenhua Jia \\ Hebei Yiling Hospital \\ Xinli Li \\ Nanjing Medical University affiliated Nanjing Hospital: Nanjing First Hospital \\ Jiangang Shen ( $\sim$ shenjg@hkucc.hku.hk) \\ University of Hong Kong https://orcid.org/0000-0003-4772-5300
}

\section{Research}

Keywords: Qiliqiangxin (QLQX) capsule, traditional Chinese medicine (TCM), TCM syndrome differentiation, syndrome, syndrome element, tongue diagnosis, syndrome questionnaire, chronic heart failure, treatment efficacy

Posted Date: July 24th, 2021

DOI: https://doi.org/10.21203/rs.3.rs-727082/v1

License: (c) (i) This work is licensed under a Creative Commons Attribution 4.0 International License. Read Full License 
1 Study Protocol: Traditional Chinese Medicine (TCM) Syndrome Differentiation for Heart

2 Failure patients and its Implication for Long-term Therapeutic Outcomes of the Qiliqiangxin

3 Capsules

5 Alice Yeuk Lan Leung ${ }^{1}$, Hoiyong $\mathrm{Chen}^{1}$, Zhenhua $\mathrm{Jia}^{2,3}$, Xinli $\mathrm{Li}^{4}$, Jiangang Shen ${ }^{1 *}$

$7 \quad{ }^{1}$ School of Chinese Medicine, University of Hong Kong, Hong SAR, P.R. China

$8{ }^{2}$ National Key Laboratory of Collateral Disease Research and Innovative Chinese Medicine, 9 Shijiazhuang, China

$10{ }^{3}$ Hebei Yiling Hospital, Key Disciplines of State Administration of TCM for Collateral Disease, 11 Shijiazhuang, China

$12{ }^{4}$ Department of Cardiology, the First Affiliated Hospital with Nanjing Medical University, Guangzhou 13 Road 300, Nanjing 210029, China

*To whom correspondence should be address:

16 Prof. Jiangang Shen: School of Chinese Medicine, The University of Hong Kong, 10 Sassoon Road,

17 Pokfulam, Hong Kong; Tel: +852-3017 6429; Fax:+852-2168 4259

18 Email: shenjg@hku.hku.hk

20 Abstract

21 Background: Syndrome differentiation is a commonly used methodology and practice in Traditional

22 Chinese Medicine (TCM) guiding the diagnosis and treatment of diseases including heart failure (HF).

23 However, previous clinical trials seldom consider the impact of syndrome patterns on the outcome

24 evaluation of TCM formulae. Qiliqiangxin (QLQX) capsule is a TCM formula with cardiotonic effect

25 to improve the cardiovascular function for heart failure with proven efficacy from well-designed

26 clinical trials. Though, there is no clinical trial with a large sample size and long assessment period

27 that considers the relationship between TCM syndrome differentiation and the treatment efficacy of 
28 QLQX. In the present study, we design a study protocol to evaluate the relationship between TCM

29 syndrome differentiation and the severity of heart failure as well as its progression. Furthermore, we

30 will evaluate the impact of the TCM syndrome patterns on the efficacy of QLQX in the outcome of

31 heart failure.

32 Methods: This is a clinical study conducted in conjunction with an ongoing clinical trial (QUEST

33 Study $^{1}$ ) to evaluate the efficacy of QLQX to improve the outcome of HF treatment through the

34 assessment of morbidity and re-hospitalization, which involves over 100 hospitals in mainland China

35 and Hong Kong SAR with 3,080 HF patients. Firstly, we will apply a modified TCM Questionnaire to

36 collect the clinical manifestations of HF and acquire the tongue images of the patients to facilitate the

37 syndrome differentiation. We will base on the "2014 Consensus from TCM experts on diagnosis and

38 treatment of chronic heart failure" to evaluate the TCM syndromes for the patients. Secondly, we will

39 investigate the characteristic of TCM syndrome distribution of HF patients and its correlation with the

40 functional and biochemical data. Furthermore, we will evaluate the relationship between the TCM

41 syndrome patterns and the efficacy of QLQX in the treatment of heart failure. Lastly, we will

42 investigate the implication of tongue diagnosis in the severity and therapeutic outcome of HF.

43 Expect Outcomes: To our knowledge, this will be the latest and well-designed clinical trial to evaluate

44 the rules of TCM syndrome differentiation in the progression and therapeutic outcome of HF patients. 
45 We expect to obtain direct clinical evidence to verify the importance of TCM syndrome differentiation

46 for the diagnosis and treatment of HF.

47

48 Trial Registration: ${ }^{1}$ The trial was registered at Chinese Clinical Trial Registry,

49 http://www.chictr.org.cn. (Registration No.: ChiCTR1900021929); Date: 2019-03-16.

50

51 Keywords: Qiliqiangxin (QLQX) capsule, traditional Chinese medicine (TCM), TCM syndrome

52 differentiation, syndrome, syndrome element, tongue diagnosis, syndrome questionnaire, chronic heart

53 failure, treatment efficacy

54 


\section{Background}

57 Heart Failure (HF) is an end-stage cardiovascular condition characterized by structural or functional

58 cardiac impairments of ventricular filling or blood ejection, leading to abnormal hemodynamics,

59 activating neuro-hormones, and developing myocardial remodeling $(1,2)$. The typical clinical

60 manifestations include shortage of breath, fatigue and ankle swelling, accompanied by peripheral

61 edema and elevated jugular venous pressure, etc. $(3,4)$. HF is one of the leading causes of death

62 globally (5). There are approximately 64.3 million patients with heart failure worldwide (6). In the US,

63 there are around 5.7 million HF patients and it is estimated to reach 8 million HF patients in 2030,

64 accounting for a $46 \%$ increase in prevalence(7). Around $30-40 \%$ of HF patients have a history of

65 hospitalization due to $\mathrm{HF}$, and the reported 5-year all-cause readmission, readmission for HF, and

66 mortality rates are $80.4 \%, 42.3 \%$, ad $75.4 \%$ respectively $(8,9)$. Thus, HF becomes a major public

67 health burden in human diseases.

69 Current therapeutic strategies for HF include the following catalogs: Using nitrates and hydralazine to

70 resolve vasoconstriction; Using angiotensin-converting enzyme (ACE) inhibitors, angiotensin II

71 receptor blockers (ARBs), beta-blockers, and mineralocorticoid antagonists (MRAs) to target neuro- 
72 hormonal systems; Using Invabradine to target the sinus nodes discharge and slow down the heart

73 rates for the HF patients with unsatisfactory results with beta-blocker; Using cardiac resynchronization

74 therapy (CRT) targeted the sinus rhythm and implantable cardioverter-defibrillator (ICD) targeted

75 patients with cardiac arrhythmia, etc. (5). Even with the advanced treatment approaches developed,

76 the outcome of HF treatments is still unsatisfactory as the re-hospitalization of worsening heart failure

77 and mortality remain high.

79 Traditional Chinese Medicine (TCM) formulae, including Qiliqiangxin, Nuanxin, Shencaotongmai,

80 Yangxinkang, appears to be the effective adjunct therapies with conventional drugs to improve cardiac

81 function of HF patients (10). According to the TCM theory of Collateral Disease, the heart-qi and/or

82 heart-yang deficiency could induce blood stagnation and the disruption of body fluid metabolism (11).

83 The HF-induced hemodynamic dysfunctions and edema could be attributed to the pathological status

84 of heart-qi deficiency accompanied with blood stagnation and body fluid accumulation (12). Syndrome

85 differentiation is a unique TCM diagnosis in guiding therapeutic strategies for heart failure. However,

86 there is no standardized methodology for syndrome differentiation of heart failure (13-15). The

87 diversities of syndromes lead to difficulties in drawing conclusive outcomes and therefore, hindering

88 the development of TCM treatment for heart failure $(16,17)$. On the other hand, Tongue Diagnosis is 
89 crucial for identifying syndromes and evaluating the therapeutic responses in TCM practice. Tongue

90 Diagnosis has been applied for different diseases such as metabolic syndrome, cancer, menstrual

91 clinical symptoms, etc. (18-21). Recent studies observed the correlation between tongue patterns and

92 risk factors of heart failure in dialysis patients (22-24). The tongue changes were also observed in heart

93 failure patients with normal ejection fraction (22-24). Whether heart failure patients have specific

94 tongue patterns remains unclear. Therefore, we design this study to explore the impacts of TCM

95 syndrome patterns and tongue features on cardiovascular functions and the therapeutic outcome of

96 heart failure.

97

98 Qiliqiangxin (QLQX) capsule is a patent TCM formula approved by China Food and Drug

99 Administration for chronic heart failure (25). QLQX capsule is extracted from 11 TCM herbs including

100 Astragali Radix, ginseng radix at rhizoma, aconite lateralis radix preparata, salvia miltiorrhizae radix

101 et rhizome, semen descurainiae lepidii, alismatis rhizome, polygonati odorati rhizome, cinnamomi

102 ramulus, carthami flos, periploca cortex, and citri reticulatae periarpium. Previous studies indicated

103 that: (1) QLQX could attenuate cardiac remodeling via activating peroxisome proliferator-activated

104 receptor $-\gamma($ PPAR- $\gamma)$ and mTOR; (2) QLQX could prevent cardiomyocytes hypertrophy via activating

105 PGC-1 $\alpha$ and downregulating MiR-199a-5p, etc. (26-30); 3) QLQX could improve ejection fraction 
106 and attenuates the left ventricular remodeling via inhibitory effect of the renin-angiotensin-aldosterone

107 system (RAAS) $(31,32)$; (4) QLQX could inhibit the myocardial inflammation and cardiomyocyte

108 apoptosis to promote the proliferation of cardiomyocyte (33). A multi-center, randomized, double-

109 blind and placebo-controlled clinical trial reported that QLQX, as an adjunct therapy, reduced the level

110 of NT-proB-type natriuretic peptide (NT-proBNP) and improved the NYHA functions, left ventricular

111 ejection fraction (LVEF), 6-min walking distance, quality of life and composite cardiac events in HF

112 patients (34). Those studies suggest that QLQX is a promising TCM formula for HF patients. However,

113 the efficacy of QLQX on hard endpoint incidents such as re-hospitalization of worsening HF and

114 mortality is yet to be proven. Particularly, whether different TCM syndrome patterns impact the

115 therapeutic outcomes of QLQX is unknown. As such, we have designed QUEST study (35) to explore

116 the long-term efficacy of QLQX in addition to conventional heart failure treatment of CHF. The

117 primary outcome will be the occurrence of the composite endpoint which is defined as either

118 cardiovascular (CV) death or re-hospitalization due to the exacerbation of HF. The secondary outcome

119 measures include the all-cause mortality, secondary endpoint events (treatment terminated due to

120 worsening heart failure, successful resuscitation after cardiac arrest, malignant arrhythmia, non-fatal

121 stroke), CV death and re-hospitalization due to worsening heart failure in patients with ischemic heart

122 disease, and the level of Serum NT-proBNP. In the present study, we target to evaluate the impacts of 
123 TCM syndrome differentiation on the disease progress of HF and the therapeutic outcome of QLQX.

124 The study is coached by three specific objectives to answer the following questions: 1) Whether TCM

125 syndromes are associated with the severity of heart failure; 2) Whether different TCM syndrome

126 patterns have different therapeutic outcomes in QLQX treatment for heart failure; and 3) Whether

127 TCM tongue diagnostic characteristics could be an effective index for the severity of heart failure and

128 the indication of the progression as well as the therapeutic outcomes.

130 Methods/Design

131 1. Patient Recruitment and Selection Criteria

132 Our study is conducted in conjunction with a multicenter, randomized, double-blind, placebo-

133 controlled, parallel-group, event-driven clinical study named the QUEST on chronic heart failure

134 patients. This study is carried out in approximately 100 centers located in China and Hong Kong SAR.

135 These centers are eligible hospitals or affiliated hospitals of the medical universities. The target number

136 of patient recruitments will be 3,080, which is calculated according to the PARADIGM-HF study

137 based on the cardiovascular death or hospitalization rate for heart failure (36). This is an event-driven

138 study and all the recruited patients will remain in the study (whether taking the study drug or not) until

139 the number of the primary endpoint events reaches the predicted target of 620 cases, or the study meets 
140 the pre-defined efficacy or safety criteria assessed by the Clinical Event Adjudication Committee

141 (CEAC).

143 Recruitment of patients is assessed according to the pre-defined inclusion and exclusion criteria of the

144 QUEST study (Table 1). Recruited patients must be at least 18 years old, can fulfill all the inclusion

145 criteria including the NT-proBNP, LVEF, NYHA cardiac functional grading, and the standardized

146 drug treatment for at least 2 weeks prior to enrollment, etc., and not meeting any exclusion criterion.

148 Table 1. Inclusion and Exclusion Criteria

Inclusion criteria:

1 ) Provision of signed informed consent prior to any study specific procedures;

2 ) Male or female, aged $\geq 18$ years at the time of consent;

3 ) Established documented diagnosis of heart failure for at least three months ago according to "Chinese Heart Failure Diagnosis and Treatment Guideline" issued by the Chinese Medical Association Cardiovascular Branch.

4 ) Left ventricular ejection fraction $(\mathrm{LVEF}) \leq 40 \%$ (echocardiogram, radionuclide, ventriculogram, contrast angiography or cardiac MRI );

5 ) NYHA cardiac functional grading II to III, with stable clinical symptoms; including those diagnosed as grade IV within 2 weeks before enrollment;

6) Serum NT-proBNP $\geq 450 \mathrm{pg} / \mathrm{ml}$;

7 ) Those who have received standardized baseline treatment regimens without doses adjusted and given intravenously for at least two weeks prior to enrollment; standardized drug treatment includes: angiotensin-converting enzyme inhibitor (ACEI) or angiotensin receptor blocker (ARB) or angiotensin receptor neprilysin inhibitor (ARNI), beta blocker, and aldosterone receptor antagonist (the optimal therapeutic dose should be achieved, except for contraindications or intolerance); 


\section{Exclusion criteria:}

Patients should not enter the study if any of the following exclusion criteria are fulfilled

1) Heart failure caused by valvular disease, congenital heart disease, pericardial disease, arrhythmia or non-cardiogenic disease, or caused by vital organ failure (such as renal, hepatic failure, etc.); and right heart failure caused by pulmonary or other definite causes; and acute heart failure;

2 ) Coronary revascularization (percutaneous coronary intervention [PCI] or coronary artery bypass grafting $[\mathrm{CABG}]$ ) or cardiac synchronization therapy planned to undergo after randomization, or had received cardiac resynchronization therapy prior to enrolment;

3 ) Any condition outside the $\mathrm{CV}$ diseases such as but not limited to malignant tumor, severe mental illness, hematopoietic diseases, neuroendocrine system disease, liver transaminase and alkaline phosphatase $\geq 3$ x upper limit of normal (ULN), abnormal renal function serum creatinine $>2$ $\mathrm{mg} / \mathrm{dl}(176.82 \mathrm{umol} / \mathrm{L})$, potassium $>5.5 \mathrm{mmol} / \mathrm{L}$;

4 ) Patient with left ventricular outflow tract obstruction, myocarditis, aortic aneurysm, aortic dissection, or obvious hemodynamic changes caused by unrepaired valve;

5 ) Cardiogenic shock, uncontrollable malignant arrhythmia, sinus or atrioventricular block at second degree type II or above without pacemaker treatment, progressive unstable angina pectoris or acute myocardial infarction;

6 ) Uncontrolled hypertension systolic blood pressure (SBP) $\geq 180 \mathrm{mmHg}$ and/or diastolic blood pressure $(\mathrm{DBP}) \geq 110 \mathrm{mmHg}$; or SBP $<90 \mathrm{mmHg}$ and/or DBP $<60 \mathrm{mmHg}$;

7 ) Participation in another clinical study with an IP during the last month prior to enrolment;

8 ) Women of child-bearing potential (i.e., those who are not chemically or surgically sterilized or who are not post-menopausal) who are not willing to use a medically accepted method of contraception that is considered reliable in the judgment of the investigator, from the time of signing the informed consent throughout the study and 4 weeks thereafter, OR women who have a positive pregnancy test at enrolment or randomization OR women who are breast-feeding;

9) Allergic constitution; known to be allergic to research drug;

10 ) Inability of the patient, in the opinion of the investigator, to understand and/or comply with study medications, procedures or any conditions may render the patient unable to complete the study.

150 Before the recruitment, patients must be "clinically stable" by receiving at least two weeks of 
152 fixed drug type and dosage, as well as have not taken any intravenous anti-HF drug nor oral TCM

153 medication with similar composition as the QLQX capsules. Once the patients are clinically stable,

154 they will be assigned to the QLQX group or the control group in a ratio of 1-to-1 through the double-

155 blind computerized randomize allocation. The patient will be given either QLQX capsules or placebo

156 capsules during the assessment period in addition to their standard treatment for heart failure following

157 the guidelines for diagnosis and treatment of HF in China 2018 (25) or local guideline. The QLQX

158 capsules and placebo are identical in size and shape. The dosage used in this study is 4 capsules of

159 QLQX or placebo 3 times daily. During the assessment period, patients should not take any TCM or

160 herbs which have similar contents to the QLQX capsule.

162 The entire study will last for approximately 36 months with the first 24 months as the recruitment

163 period. The study will be terminated once the last patient has finished the 12-months assessment. Once

164 recruited, the patient is required to visit the hospitals for efficacy and safety assessment according to

165 the study schedule. All patients are required to sign the Informed Consent Form before the recruitment.

166 For each visit, the patient will be assessed by the researchers/physician. A range of clinical information

167 and parameters will be collected and tests will be performed for the assessment of TCM syndrome, the

168 treatment efficacy, and safety including laboratory and ECG results, etc. The assessment of safety of 
172 respectively) are gathered. Patients are allowed to withdraw from the study for any reason but the

173 reason is required to be recorded in the case report form. If the patient has an intolerable adverse event

174 that is related to the study drug based on the researcher's judgment, the patient should immediately

175 discontinue the treatment with the study drug. The study flowchart is shown in Fig. 1.

\section{Enrollment}

\section{Allocation}

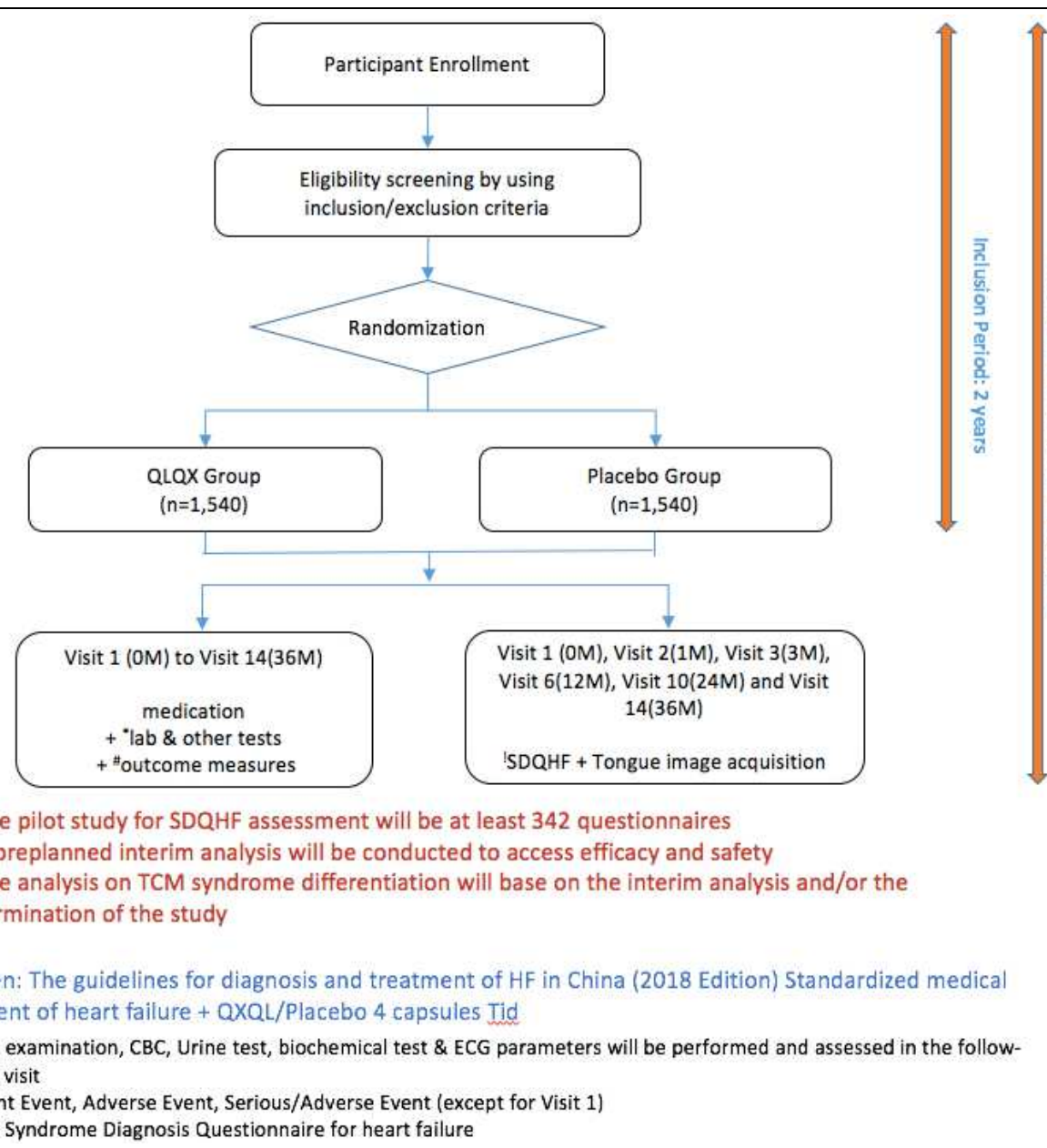

Fig. 1 Study Flow Chart 
180 2. TCM Syndrome Diagnosis Questionnaire for Heart Failure (SDQHF)

181 To collect clinical information for TCM syndrome differentiation, a specialized TCM Syndrome

182 Diagnosis Questionnaire for Heart Failure (SDQHF) is developed according to the TCM syndrome

183 scales for heart failure reported by previous studies (37-40). The clinical manifestation including the

184 signs and symptoms of heart failure diagnosis which are described according to the "2012 Guiding

185 Principles for Clinical Study on New Drug for Use in Traditional Chinese Medicine", "2014

186 Consensus from TCM experts on diagnosis and treatment of chronic heart failure", "2017 Guideline

187 for TCM Diagnosis and Treatment of Heart Failure (Chronic Heart Failure)” (16, 41, 42).

189 In the SDQHF, there are 36 closed-ended items and each item is given a ranked scale with simple

190 descriptions to indicate the features of the severity or frequency of signs or symptoms. These 36 items

191 are carefully considered to incorporate the major and concomitant signs/symptoms which are essential

192 for the diagnosis of HF and the TCM syndrome differentiation. The descriptive scale per item is clearly

193 defined and the scales were input by a group of experienced clinical experts and physicians. The

194 SDQHF has gone through the review and pre-pilot testing by 20 subject matter experts including

195 physicians and TCM practitioners. 
197 The 36-items are divided into several dimensions including major signs and symptoms of heart failure,

198 cold and heat characteristics, perspiration, head and general body condition, diet, etc. to facilitate the

199 differentiation of heart failure and TCM syndrome. The terminologies used are specially considered

200 to avoid jargon so that the questionnaire can be easily understood by the researchers/physicians and

201 the patients. It will be an interviewer-administered questionnaire so that unclear questions can be

202 clarified to the patients and ensure patients answer all the questions. The first 31 items in the SDQHF

203 are required to be answered by the patients, and the last 5 items about the physical signs of the patients

204 should be determined by the physician/researcher based on their observation of the patients. The list

205 of 36-items in SDQHF is shown in Table 1.

206

207

Table 1. The list of 36-items in the TCM Symptom Diagnosis Questionnaire for Heart Failure 209 (SDQHF)

\begin{tabular}{|l|l|l|l|}
\hline 1. Palpitations & 10. Coughing out of Phlegm & 19. Cold Sweat & 28. Constipation \\
\hline 2. Chest Tightness & 11. Color of phlegm & 20. Fear of cold & 29. Diarrhea \\
\hline 3. Precordial pain & 12. Texture of phlegm & 21. Coldness feeling in upper & 30. Frequency of urination \\
\hline 4. Dizziness & 13. Feeling fullness in & 22. Feeling heat in 5 centers & 31. Amount of urination \\
\hline 5. Shortness of breath & $\begin{array}{l}\text { 14. Hypochondriac pain or } \\
\text { feeling of distension }\end{array}$ & 23. Appetite & \\
\hline 6. Gasp for breath & 15. Edema & 32. Distension of jugular vein \\
\hline 7. Posture & 16. Tiredness & 24. Sense of thirstiness & 33. Cyanosis of face and lips \\
\hline 8. Coughing & 25. Loss of sleep/insomnia & 34. Color of face/complexion \\
\hline
\end{tabular}


9. Phlegm in throat with sound
18. Sweating at night time

211 All enrolled patients are required to complete this questionnaire in a total of 6 specific study visits to

212 the hospitals (i.e. Visit 1 at (Day 0), Visit 2(1M), Visit 3(3M), Visit 6(12M), Visit 10(24M) and Visit

213 14(36M)/EOS). The visit schedule and list of parameters are shown in Table 2. For each scheduled

214 visit, the physician/researcher will conduct a face-to-face interview with the patient to go through this

215 survey questionnaire. The entire interview process will last for approximately 8 to 10 minutes. The

216 data collected will be first recorded manually in the paper SDQHF and then input into the Epidata

217 software(43) which is the centralized database for the collection of research data from the QUEST

218 study.

219

220 Table 2. Planned visits and parameters

\begin{tabular}{|c|c|c|c|c|c|c|c|c|c|c|c|c|c|c|c|c|}
\hline \multirow[b]{2}{*}{ Visit Schedule } & \multirow{2}{*}{$\begin{array}{c}\text { Enrollment \& } \\
\text { Allocation } \\
1\end{array}$} & \multicolumn{13}{|c|}{ Postallocation } & \multicolumn{2}{|c|}{ Closeout } \\
\hline & & 2 & 3 & 4 & 5 & 6 & 7 & 8 & 9 & 10 & 11 & 12 & 13 & 14 & UNS & EOS \\
\hline \multirow[t]{2}{*}{ Day/Month } & Day0 & $1 \mathrm{M}$ & $3 \mathrm{M}$ & $6 \mathrm{M}$ & $9 \mathrm{M}$ & $12 \mathrm{M}$ & $15 \mathrm{M}$ & $18 \mathrm{M}$ & $21 \mathrm{M}$ & $24 \mathrm{M}$ & $27 \mathrm{M}$ & $30 \mathrm{M}$ & $33 \mathrm{M}$ & $36 \mathrm{M}$ & & \\
\hline & -14 & \pm 3 & \pm 7 & \pm 7 & \pm 7 & \pm 7 & \pm 7 & \pm 7 & \pm 7 & \pm 7 & \pm 7 & \pm 7 & \pm 7 & \pm 7 & & $=2 \mathrm{wks}$ \\
\hline Informed Consent & $\mathrm{x}$ & & & & & & & & & & & & & & & \\
\hline Inclusion/Exclusion criteria & $\mathrm{x}$ & & & & & & & & & & & & & & & \\
\hline Randomization & $\mathrm{x}$ & & & & & & & & & & & & & & & \\
\hline General data and medical history & $\mathrm{x}$ & & & & & & & & & & & & & & & \\
\hline Medical history of HF & $\mathrm{x}$ & & & & & & & & & & & & & & & \\
\hline Medical history of CV Diseases & $\mathrm{x}$ & & & & & & & & & & & & & & & \\
\hline Physical examination & $\mathrm{x}$ & $x^{1}$ & $x^{1}$ & $x^{1}$ & $x^{1}$ & $\mathrm{x}$ & $x^{1}$ & $x^{1}$ & $x^{1}$ & $\mathrm{x}$ & $x^{1}$ & $x^{1}$ & $x^{1}$ & $\mathrm{x}$ & (x) & $\mathrm{x}$ \\
\hline
\end{tabular}




\begin{tabular}{|c|c|c|c|c|c|c|c|c|c|c|c|c|c|c|c|c|}
\hline Heart failure medications & $\mathrm{x}$ & $\mathrm{x}$ & $\mathrm{x}$ & $\mathrm{x}$ & $\mathrm{x}$ & $\mathrm{x}$ & $\mathrm{x}$ & $\mathrm{x}$ & $\mathrm{x}$ & $\mathrm{x}$ & $\mathrm{x}$ & $\mathrm{x}$ & $\mathrm{x}$ & $\mathrm{x}$ & (x) & $\mathrm{x}$ \\
\hline Medications of other CVD & $\mathrm{x}$ & $\mathrm{x}$ & $\mathrm{x}$ & $\mathrm{x}$ & $\mathrm{x}$ & $\mathrm{x}$ & $\mathrm{x}$ & $\mathrm{x}$ & $\mathrm{x}$ & $\mathrm{x}$ & $\mathrm{x}$ & $\mathrm{x}$ & $\mathrm{x}$ & $\mathrm{x}$ & (x) & $\mathrm{x}$ \\
\hline Other medications & $\mathrm{x}$ & $\mathrm{x}$ & $\mathrm{x}$ & $\mathrm{x}$ & $\mathrm{x}$ & $\mathrm{x}$ & $\mathrm{x}$ & $\mathrm{x}$ & $\mathrm{x}$ & $\mathrm{x}$ & $\mathrm{x}$ & $\mathrm{x}$ & $\mathrm{x}$ & $\mathrm{x}$ & (x) & $\mathrm{x}$ \\
\hline TCM Syndrome Diagnosis & $\mathrm{x}$ & $\mathrm{x}$ & $\mathrm{x}$ & & & $\mathrm{x}$ & & & & $\mathrm{x}$ & & & & $\mathrm{x}$ & & $\mathrm{x}$ \\
\hline Questionnaire (SDQHF) & & & & & & & & & & & & & & & & \\
\hline Tongue Image Acquisition & $\mathrm{x}$ & $\mathrm{x}$ & $\mathrm{x}$ & & & $\mathrm{x}$ & & & & $\mathrm{x}$ & & & & $\mathrm{x}$ & & $\mathrm{x}$ \\
\hline Echocardiogram & $\mathrm{x}^{*}$ & & & & & & & & & & & & & & & \\
\hline Pregnancy Tests & $\mathrm{x}$ & & & & & $\mathrm{x}$ & & & & $\mathrm{x}$ & & & & $\mathrm{x}$ & (x) & $\mathrm{x}$ \\
\hline Blood/urine routine test & $\mathrm{x}$ & $\mathrm{x}$ & & & & $\mathrm{x}$ & & & & $\mathrm{x}$ & & & & $\mathrm{x}$ & (x) & $\mathrm{x}$ \\
\hline Biochemical test & $\mathrm{x}$ & $\mathrm{x}$ & & & & $\mathrm{x}$ & & & & $\mathrm{x}$ & & & & $\mathrm{x}$ & (x) & $\mathrm{x}$ \\
\hline 12-lead ECG & $\mathrm{x}^{*}$ & $\mathrm{x}$ & & & & $\mathrm{x}$ & & & & $\mathrm{x}$ & & & & $\mathrm{x}$ & $(\mathrm{x})$ & $\mathrm{x}$ \\
\hline Serum NT-proBNP at local & $\mathrm{x}$ & $\mathrm{x}$ & & $\mathrm{x}$ & & & & & & & & & & & & \\
\hline laboratory & & & & & & & & & & & & & & & & \\
\hline Dispensing IP & $\mathrm{x}$ & & $\mathrm{x}$ & $\mathrm{x}$ & $\mathrm{x}$ & $\mathrm{x}$ & $\mathrm{x}$ & $\mathrm{x}$ & $\mathrm{x}$ & $\mathrm{x}$ & $\mathrm{x}$ & $\mathrm{x}$ & $\mathrm{x}$ & $\mathrm{x}$ & & \\
\hline Returning IP for accountability & & & $\mathrm{x}$ & $\mathrm{x}$ & $\mathrm{x}$ & $\mathrm{x}$ & $\mathrm{x}$ & $\mathrm{x}$ & $\mathrm{x}$ & $\mathrm{x}$ & $\mathrm{x}$ & $\mathrm{x}$ & $\mathrm{x}$ & $\mathrm{x}$ & & $\mathrm{x}$ \\
\hline Endpoint Event & & $\mathrm{x}$ & $\mathrm{x}$ & $\mathrm{x}$ & $\mathrm{x}$ & $\mathrm{x}$ & $\mathrm{x}$ & $\mathrm{x}$ & $\mathrm{x}$ & $\mathrm{x}$ & $\mathrm{x}$ & $\mathrm{x}$ & $\mathrm{x}$ & $\mathrm{x}$ & (x) & $\mathrm{x}$ \\
\hline Adverse Event & & $\mathrm{x}$ & $\mathrm{x}$ & $\mathrm{x}$ & $\mathrm{x}$ & $\mathrm{x}$ & $\mathrm{x}$ & $\mathrm{x}$ & $\mathrm{x}$ & $\mathrm{x}$ & $\mathrm{x}$ & $\mathrm{x}$ & $\mathrm{x}$ & $\mathrm{x}$ & (x) & $\mathrm{x}$ \\
\hline Serious Adverse Event & & $\mathrm{x}$ & $\mathrm{x}$ & $\mathrm{x}$ & $\mathrm{x}$ & $\mathrm{x}$ & $\mathrm{x}$ & $\mathrm{x}$ & $\mathrm{x}$ & $\mathrm{x}$ & $\mathrm{x}$ & $\mathrm{x}$ & $\mathrm{x}$ & $\mathrm{x}$ & (x) & $\mathrm{x}$ \\
\hline
\end{tabular}

$221 \cdot \mathrm{X}^{1}$ Simplified physical examination

$222 \cdot x^{*}$ Cardiac ultrasound and 12-lead ECG within the first 6 months of enrollment

223 - UNS (unplanned visit): (x) The marked item is optional and performed according to the judgment of researchers

224 - EOS (final visit): Make arrangement according to the study end time (if there is a visit within one month before the end of study, it is

225 regarded as a final visit, but needs to be supplemented with the items required completely)

226 - Pregnancy test is only applicable to women of childbearing age (if the urine pregnancy test is positive, it must be confirmed by serum 227 pregnancy test).

\section{3. TCM syndrome differentiation}

230 According to the "2014 Consensus from TCM experts on diagnosis and treatment of chronic heart

231 failure" (17), there are 3 major types of TCM syndrome for heart failure: 1) qi-deficiency with blood

232 stasis syndrome; 2) qi-yin deficiency with blood stasis syndrome; 3) yang-qi deficiency with blood 
233 stasis syndrome, and each can be associated with the phlegm-retention syndrome. These syndromes

234 are comprised of a combination of 2-4 syndrome elements including 1) qi-deficiency; 2) yin-deficiency,

235 3) yang-deficiency; 4) blood stasis and 5) phlegm-retention. The list of clinical manifestations

236 including signs and symptoms, pulse, and tongue features of the 3 TCM syndrome types are shown in

237 Fig. 2. The 5 syndrome elements with 3 syndrome types indicated in this guideline are impeccably

238 matched with the TCM Theory of Collateral Disease for heart failure.

Fig. 2 Diagnosis and TCM Syndrome Differentiation for Heart Failure according to the "2014

246 A scoring model for syndrome differentiation is developed with reference to the criteria listed in Fig.2

247 and the TCM theories. The output from the scoring model will be the type of TCM syndrome and the 
248 level of severity of heart failure patients at a specific stage of the disease. The output will be used for

249 further analysis on the correlation of syndrome differentiation to the biomedical indicators, the severity

250 of HF, and the therapeutic outcome of QLQX capsules.

252 4. Correlation analysis of TCM syndrome patterns and the therapeutic outcome of QLQX in 253 CHF

254 Our primary objective is to explore the implication of TCM syndrome to the severity of heart failure 255 and the therapeutic outcome of QLQX capsules. We hypothesize that the efficacy of QLQX Capsule 256 on heart failure could be related to the clinical syndrome patterns in TCM diagnosis and likely be 257 favorable to the patients with qi-deficiency with blood stasis and yang-qi deficiency with blood stasis.

259 The efficacy of QLQX capsule will be evaluated with reference to the "2012 Guiding Principles for

260 Clinical Study on New Drug for Use in Traditional Chinese Medicine". We will evaluate the

261 correlation between TCM syndrome patterns and the severity of heart failure the re-hospitalization

262 rates and mortality. The distribution of TCM syndrome will also be evaluated by NYHA classification,

263 results from an electrocardiogram (ECG), cardiac ultrasound interpretation, and biochemical

264 parameters from routine blood tesst such as NT-proBNP, urine test, etc. Other outcomes include: 1) 
265 the distribution of TCM syndrome patterns in heart failure patients; 2) the correlation between TCM

266 syndrome patterns and the severity of heart failure and 3) the dynamic changes between biomedical

267 parameters and the TCM syndrome patterns of heart failure, etc.

\section{5. Correlation of tongue features and the severity of HF and therapeutic outcome of QLQX}

270 Tongue images of the patients will be acquired as part of the TCM Syndrome Diagnosis Questionnaire

271 for Heart Failure (SDQHF) during the study visit. To ensure the quality and consistency of tongue

272 photos from all clinical centers/hospitals, all researchers/physicians are properly trained according to

273 the Standard Operation Procedures (SOP), and are provided with a standardized image acquisition

274 device to capture the tongue image. The SOP includes the schedule of the study visit, the procedures

275 for completing the SDQHF, and the criteria for capturing the tongue image such as the lighting

276 requirement, camera mode (i.e. normal mode, no flashes, etc.), the posture of the tongue, and the

277 tongue area to be included in the image, as well as the prerequisite requirements such as no intake of

278 food or drinks that will stain the tongue coating on the day of the study visit. All tongue images should

279 be sent to the central tongue image database via email with an indication of the patient number and the

280 date of study visit. 
282 We will set up a team of 4 TCM practitioners with 3 to 30 years of clinical experience to evaluate the

283 tongue images. The identifiers and possible values for tongue analysis include the tongue color, tongue

284 shape, tongue coating proper and its color, tongue coating distribution, appearance and location of

285 ecchymosis, etc. Tongue images that fail to meet the SOP requirement will be excluded from the

286 analysis. A 4-stage decision flow is designed for the review and analysis of tongue images: 1) direct

287 confirmation if consistent interpretation and diagnosis from the first three TCM practitioners; 2) if

288 consistent results are identical from 2 TCM practitioners but different diagnosis from the 3rd one, take

289 the result from the majority; 3) if the interpretation and diagnostic results from all 3 TCM practitioners

290 are different, will involve the 4th TCM practitioner with over 30 years of clinical experience to judge

291 the syndrome type with the clinical information collected from the SDQHF; and finally, 4) if the image

292 is determined as not in good quality for drawing the conclusion after reviewed by the separate TCM

293 practitioner and professor, that tongue image will be excluded from the study.

294 Outcomes of the tongue analysis include 1) determination of the TCM syndrome differentiation of

295 patients throughout the assessment period with the clinical information collected through the SDQHF;

296 2) tongue features in heart failure patients in accordance to the TCM syndrome, NYHA classification,

297 and other biomedical data; 3) dynamic patterns of tongue condition in relation to the treatment efficacy

298 and other biomedical indicators 


\section{Data Management}

300 The patient's demographic data and all clinical data will be recorded and kept in the physical medical

301 record folder during each study visit. The data will then be input into the Epidata software for

302 centralization of all research data and all the tongue images will be kept in a separate centralized image

303 database. The data management process will be complied with the regulatory requirements of Clinical

304 Trial Quality Management Regulations and Clinical Trial Data Management Work Technical

305 Guidelines to ensure the authenticity, integrality, accuracy, and traceability of data.

\section{7. Sample size estimations}

308 This study is benchmarked with the QUEST study in which 3080 patients will be enrolled in over 100

309 centers (1540 patients per group) and followed up for at least 12 months. The sample size was

310 estimated referring to the PARADIGM-HF study (44). For QUEST study, the estimated incidence of

311 cardiovascular death and hospitalization for heart failure is $25 \%$ in all patients receiving basic

312 treatment + placebo group within 36 months of follow-up and $20 \%$ with those receiving basic

313 treatment + QLQX Capsule group. The random distribution ratio is 1:1 between the study group and

314 the control group. With the consumption of type I error in the interim analysis, $\alpha$ is adjusted to

315 unilateral 0.02314 . The sample size is the number of cases with composite endpoints events. Thus, 620 
316 composite endpoint events are expected for the QUEST study to provide $80 \%$ power of test $(\beta=0.2)$,

317 and the $20 \%$ risk can be reduced in the study group by the log-rank test.

318 For the current study, a pilot test with at least 342 patients will be conducted to evaluate the

319 acceptability, reliability, and validity of the questionnaire. The number of patients for pilot testing is

320 calculated based on the confidence level of $95 \%$, population size of 3,080 , and $5 \%$ margin of error

321 for this 36-item questionnaire.

\section{8. Data analysis and statistic methodology}

324 Analysis of the TCM Questionnaire will be performed with the SPSS statistical software package,

325 version SPSS 24.0 (Chicago, IL,USA). Data will be presented by Mean \pm S.D. Principal Component

326 Analysis (PCA) will be applied to the questionnaire to examine the factor structure. Logistic regression

327 analysis will be used to evaluate the capability of SDQHF to diagnose the severity of HF from a

328 functional perspective. Pearson's correlation coefficient will be used to determine the existence of

329 correlations between TCM syndrome differentiation and various parameters such as treatment efficacy,

330 NT-proBNP, NYHA classification, etc. An independent t-test will be used to compare quantitative

331 variables for the two-group designed study. Fisher's Exact test and the chi-squared test will be applied 
332 to compare categorical variables between groups. Multiple groups designed data will be analyzed by

333 two-way ANOVA.

\section{Discussion}

336 In TCM, the syndrome pattern is a vital component in both diagnosis and treatment of disease.

337 Syndrome elements, syndromes, phenotypic features, as well as disease, form an integral process in

338 the diagnostic path. However, due to the lack of standardization of syndromes, many diversified

339 syndrome types were found in previous studies, leading to difficulties in drawing sound conclusive

340 outcomes and thus, hindering research development (13-17). It is crucial to have an end-to-end

341 approach with a clear and definitive set of syndromes for heart failure study. The present study will

342 focus on the objective and quantitative syndrome diagnosis criteria for heart failure, the scoring model

343 of the SDQHF, and the establishment of the tongue image database. For the TCM syndrome pattern

344 recognition, we aim to develop a scoring system to catalog the syndrome differentiation from the signs

345 and symptoms in the questionnaire with reference to the guideline and consensus including the "2012

346 Guiding Principles for Clinical Study on New Drug for Use in Traditional Chinese Medicine”, "2014

347 Consensus from TCM experts on diagnosis and treatment of chronic heart failure", and "2017

348 Guideline for TCM Diagnosis and Treatment of Heart Failure (Chronic Heart Failure)”. We expect the 
349 development of SDQHF and the scoring model could establish a solid ground for syndrome

350 differentiation of heart failure and to facilitate the evaluation of the efficacy of the QLQX Capsule.

351 With the large-scale clinical trial and multiple level evaluations, we could provide an example to

352 resolve the unanswered question of whether the evidence-based clinical trials should consider the

353 potential influence on different TCM syndrome differentiation patterns on disease progress and

354 therapeutic outcome. This may enable the paradigm shift from reactive to predictive, preventive, and

355 personalized care through the application of the TCM syndrome differentiation.

356 In terms of operational issues, quality control is one of the major challenges for such large-scale

357 clinical studies involving over 100 clinical centers and more than 300 researchers/physicians in China

358 and Hong Kong. We have established standardized operating procedure (SOP) and provided extensive

359 training to all researchers/physicians prior to the patient recruitments. With the onset, supplementary

360 training and continuous review, we could ensure proper execution of SOP to secure the quality of data

361 collection. Tongue image acquisition is one of the critical issues for quality control. As such, we

362 provided an imaging capturing device of the same model to each clinical center and trained all the

363 researchers/physicians on how to properly acquire the tongue image, including the camera settings,

364 the lighting requirement, and other pre-requisite requirements for patients prior to the photo-taking,

365 etc. To ensure the image quality, we have a constant quality check on all tongue photos so that feedback 
366 for improvement could be provided to the researchers/physicians on a timely basis. Thus, the quality

367 of tongue images could meet our requirement for the analysis of tongue features and patterns of heart

368 failure. We will also put efforts into patient communication to ensure all patients have a thorough

369 understanding of the trial so that they can strictly follow through the requirement and attend all

370 scheduled visits. With the high standard and high-quality clinical trial, we expect to achieve following

371 outcomes: (1) To verify that QLQX could be an adjunct therapy with conventional treatment to reduce

372 the mortality and re-hospitalization with worsening heart failure in the QUEST study. (2) To confirm

373 whether different TCM syndrome patterns and tongue characteristics would be associated with the

374 severity of heart failure and the therapeutic efficacy of QLQX on chronic heart failure patients.

\section{Conclusion}

377 To our knowledge, this study is a high standard designed, multi-center, randomized, double-blind,

378 parallel-group, placebo-controlled clinical trial with large scale subjects to verify the impacts of TCM

379 syndrome patterns on the severity of heart failure and the therapeutic outcome of Chinese medicine

380 formula. Furthermore, it may very well be the largest cohort clinical trial with long follow-up period

381 and hard endpoints to evaluate the long-term efficacy and safety of TCM formula in the treatment of

382 chronic heart failure.

$384 \quad$ Trial status 
385 At the time of manuscript submission, the patient recruitment for the trial has just finished and we are

386 now in the assessment period.

387

388 List of abbreviations

389 6MWD: 6-min Walking Distance, CHF: chronic heart failure, HF: heart failure, NYHA: New York

390 Heart Association; QLQX: Qiliqiangxin Capsule, LVMi: Left ventricular mass index, LVEF: Left

391 ventricular ejection fraction, TCM: Traditional Chinese Medicine; SDQHF: Syndrome Diagnosis

392 Questionnaire for heart failure, NT-proBNP: NT-proB-type Natriuertic Peptide; SOP: Standard 393 Operating Procedures

394

395 


\section{Ethics approval and consent to participate}

398 All participating centers must obtain approval from appropriate independent ethics committees or 399 institutional review boards (Approved No. of ethic committee:2018-SR-275). The study is conducted 400 in accordance with the principles of Good Clinical Practice and the Declaration of Helsinki. This study 401 is conducted in conjunction with a current trial which is registered at http://www.chictr.org.cn402 ChiCTR1900021929. All participants must give written informed consent which should be acquired 403 after a sufficient and detailed explanation to the research situation (including confidentiality, risk and 404 benefits, the purpose of the study, etc.). For Hong Kong, the clinical trial is approved by the 405 Department of Health with Certificate For Clinical Trial and Medicinal Test (Certificate No. CTMT406 00008).

408 Consent for publication

409 Not applicable.

411 Availability of data and materials

412 Not applicable.

$414 \quad$ Funding

415 This study is conducted in conjunction with a current study (QUEST). QUEST is funded by National 416 Key Technologies R\&D Program, Modernization of Traditional Chinese Medicine of Ministry of 417 Science and Technology of People's Republic of China (Project No. 2017YFC1700501, 418 2017YFC1700505) and the Major Program of the National Natural Science Foundation of China 419 (81730106).

420 Qiliangxin capsules and the matching placebos are provided by Shijiazhuang Yiling Pharmac eutical 421 Co., Ltd. (Shijazhuang, People's Republic of China).

422 The funding sources have not involved in the study design, data collection, analysis, interpretation of 423 data and writing the manuscript.

\section{Competing interest}

426 The design of the study, data collection, data analysis and interpretation are independent to the funding 427 body.

428 All executive committee members of QUEST study have received consulting fees as well as travel and 429 research support in the planning and conduct of the QUEST study. The authors declare that they have 430 no other conflict of interest to disclose. 


\section{Author's contribution}

433 YLL participated in the design of the research protocol and drafted the manuscript. JJS contributed to 434 the conception and design of the research protocols. JJS also performed a critical revision of the 435 manuscript for important intellectual content. HYC provided major revisions to the manuscript. ZHJ 436 contributed to obtaining funding and the contact with multi-center. LXX contributed to the conception, 437 design of the research protocols of the QUEST study. All authors read and approved the final 438 manuscript.

439

\section{Acknowledgements}

441 We gratefully acknowledge the contributions of the sites and all participants in this study. We also 442 thank Mr. Gao Wei for managing the QUEST study and provides support on all the logistics. 
447 1. Yancy CW, Jessup M, Bozkurt B, Butler J, Casey JDE, Drazner MH, et al. 2013 ACCF/AHA

448 guideline for the management of heart failure: a report of the American College of Cardiology

449 Foundation/American Heart Association Task Force on Practice Guidelines. J Am Coll Cardiol.

$450 \quad 2013 ; 62(16): \mathrm{e} 147-\mathrm{e} 239$.

451 2. Ambrosy AP, Fonarow GC, Gheorghiade M, Butler J, Chioncel O, Greene SJ, et al. The Global 452 Health and Economic Burden of Hospitalizations for Heart Failure: Lessons Learned From 453 Hospitalized Heart Failure Registries. J Am Coll Cardiol. 2014;63(12):1123-33.

454 3. Joseph P, Leong D, McKee M, Anand SS, Schwalm JD, Teo K, et al. Reducing the Global 455 Burden of Cardiovascular Disease, Part 1: The Epidemiology and Risk Factors. Circ Res. $456 \quad 2017 ; 121(6): 677-94$.

457 4. Ponikowski P, Voors AA, Anker SD, Bueno H, Cleland JGF, Coats AJS, et al. 2016 ESC 458 Guidelines for the diagnosis and treatment of acute and chronic heart failure. Kardiologia polska 459 (1957). 2016;74(10):1037-147.

460 5. Pellicori P, Khan MJ, Graham FJ, Cleland JGF. New perspectives and future directions in the 461 treatment of heart failure. Heart Fail Rev. 2020;25(1):147-59.

462 6. Groenewegen A, Rutten FH, Mosterd A, Hoes AW. Epidemiology of heart failure. Eur J Heart 463 Fail. 2020;22(8):1342-56.

$4647 . \quad$ Savarese G, Lund LH. Global Public Health Burden of Heart Failure. Card Fail Rev. $465 \quad 2017 ; 3(1): 7-11$.

466 8. Urbich M, Globe G, Pantiri K, Heisen M, Bennison C, Wirtz HS, et al. A Systematic Review of 467 Medical Costs Associated with Heart Failure in the USA (2014-2020). PharmacoEconomics. 468 2020;38(11):1219-36.

469 9. Cook C, Cole G, Asaria P, Jabbour R, Francis DP. The annual global economic burden of heart 470 failure. Int J Cardiol. 2014;171(3):368-76.

471 10. Hao P, Jiang F, Cheng J, Ma L, Zhang Y, Zhao Y. Traditional Chinese Medicine for 472 Cardiovascular Disease

473 Evidence and Potential Mechanisms. Journal of the American College of Cardiology.

$474 \quad 2017 ; 69(24): 2952-66$.

475 11. Wu Y-l. Collateral Disease Theory in Practice: People's Medical Publishing House; 2008.

476 12. Mentz RJ, O'Connor CM. Pathophysiology and clinical evaluation of acute heart failure. Nat 477 Rev Cardiol. 2016;13(1):28-35.

478 13. Yang M, Cao X. Research Progress on Objective Indexes of TCM Syndrome Types of 479 Congestive Heart Failure. Modern Journal of Integrated Chinese Traditional and Western Medicine. $480 \quad 2011 ; 20(10): 1286-8$. 

of Chronic Heart Failure Syndrome Changes in the Past 20 Years. Lishizhen Medicine and Materia Medica Research. 2015;26(11):2784-6.

484 15. Yang J. Literature Research on the TCM Syndrome Patterns of Chronic Heart Failure in Recent 48510 Year. Continuing Medical Education. 2017;31(11):152-4.

486 16. Zheng X. Guiding Pricinples for Traditional Chinese Medicine and New Medicine on Clinical 487 Trials. 1 ed. Beijing: China Medical Science Press (Beijing); 2002.

488 17. Guideline for Diagnosis and Treatment of Chronic Heart Failure. Journal of Traditional Chinese 489 Medicine. 2014;55(14):1258-60.

490 18. Kawanabe T, Kamarudin ND, Ooi CY, Kobayashi F, Mi X, Sekine M, et al. Quantification of 491 tongue colour using machine learning in Kampo medicine. European journal of integrative medicine. 492 2016;8(6):932-41.

493 19. Han S, Yang XI, Qi Q, Pan Y, Chen Y, Shen J, et al. Potential screening and early diagnosis 494 method for cancer: Tongue diagnosis. International journal of oncology. 2016;48(6):2257-64.

495 20. Lee T-C, Lo L-C, Wu F-C. Traditional Chinese Medicine for Metabolic Syndrome via TCM 496 Pattern Differentiation: Tongue Diagnosis for Predictor. Evid Based Complement Alternat Med. 497 2016;2016:1971295-8.

498 21. Tim Hideaki T. Tongue diagnosis relationship between sublingual tongue morphology in three 499 tongue protrusion angles and menstrual clinical symptoms. Journal of Chinese Integrative Medicine. $500 \quad 2015 ; 13(4): 248-56$.

501 22. WANG X, XIAO YR, Chuanyun; CAO P, LIU Y, YANG S, YIN D, et al. Correlation analysis 502 between the distribution of tongue picture and some risk factors of heart failure in dialysis patients. 503 中国医药导报. 2018;15(10):110-3.

504 23. Xu S. Observation of tongue picture and its formation mechanism in 60 patients with heart 505 failure with normal ejection fraction. 亚太传统医药. 2017;13(8):112-4.

506 24. Li D. Correspondence between petechiae per unit area of tongue surface, sublingual vein 507 thickness and heart function grade of chronic heart failure: clinical observation of 310 cases. The 508 Sixth Cardiovascular Academic Exchange Meeting of Integrated Traditional Chinese and Western 509 Medicine in Jiangxi Province; Nan Chang2013.

510 25. Association HFGoCSoCoCM, Association CHFAoCMD, Cardiology EBoCJo. Chinese Clinical 511 Guideline for Heart Failure Management 2018. Zhounghua Xin Xue Guan Bing Za Zhi.

512 2018;10(46):760-89.

513 26. Tao L, Shen S, Fu S, Fang H, Wang X, Das S, et al. Traditional Chinese Medication

514 Qiliqiangxin attenuates cardiac remodeling after acute myocardial infarction in mice. Scientific 515 reports. 2015;5(1):8374-. 
27. Zhang H, Li S, Zhou Q, Sun Q, Shen S, Zhou Y, et al. Qiliqiangxin Attenuates PhenylephrineInduced Cardiac Hypertrophy through Downregulation of MiR-199a-5p. Cell Physiol Biochem. 2016;38(5):1743-51.

28. Zhou Y, Fang H, Lin S, Shen S, Tao L, Xiao J, et al. Qiliqiangxin Protects Against Cardiac

520 Ischemia-Reperfusion Injury via Activation of the mTOR Pathway. Cell Physiol Biochem.

$521 \quad 2015 ; 37(2): 454-64$.

522 29. Lin S, Wu X, Tao L, Bei Y, Zhang H, Zhou Y, et al. The Metabolic Effects of Traditional

523 Chinese Medication Qiliqiangxin on H9C2 Cardiomyocytes. Cell Physiol Biochem.

524 2015;37(6):2246-56.

525 30. Zhang J, Huang M, Shen S, Wu X, Wu X, Lv P, et al. Qiliqiangxin attenuates isoproterenol526 induced cardiac remodeling in mice. Am J Transl Res. 2017;9(12):5585-93.

527 31. Zhang F, Zhang Y, Li X, Zhang S, Zhu M, Du W, et al. Research on Q-markers of Qiliqiangxin 528 capsule for chronic heart failure treatment based on pharmacokinetics and pharmacodynamics 529 association. Phytomedicine. 2018;44:220-30.

530 32. Li F, Wang J, Song Y, Shen D, Zhao Y, Li C, et al. Qiliqiangxin alleviates Ang II-induced 531 CMECs apoptosis by downregulating autophagy via the ErbB2-AKT-FoxO3a axis. Life Sci. $532 \quad 2021 ; 273: 119239$.

533 33. Zou Y, Lin L, Ye Y, Wei J, Zhou N, Liang Y, et al. Qiliqiangxin Inhibits the Development of 534 Cardiac Hypertrophy, Remodeling, and Dysfunction During 4 Weeks of Pressure Overload in Mice. 535 J CARDIOVASC PHARM. 2012;59(3):268-80.

34. Li X, Zhang J, Huang J, Ma A, Yang J, Li W, et al. A Multicenter, Randomized, Double-Blind, Parallel-Group, Placebo-Controlled Study of the Effects of Qili Qiangxin Capsules in Patients With Chronic Heart Failure. J Am Coll Cardiol. 2013;62(12):1065-72.

35. Yao W, Cheang I, Liao S, Zhou Y, Zhou F, Xu D, et al. Study protocol for a randomized controlled trial: Qiliqiangxin in heart failUre: assESsment of reduction in morTality (QUEST). BMC Complement Med Ther. 2020;20(1):38-.

36. McMurray JJV, Packer M, Desai AS, Gong J, Lefkowitz MP, Rizkala AR, et al. Angiotensinâ $\square$ "Neprilysin Inhibition versus Enalapril in Heart Failure. N Engl J Med.

545 37. Zhou H, Li L, Zhao H, Wang Y, Du J, Zhang P, et al. A Large-Scale, Multi-Center Urine 546 Biomarkers Identification of Coronary Heart Disease in TCM Syndrome Differentiation. J Proteome 547 Res. 2019;18(5):1994-2003.

548 38. Li X, Weil L, Jiangcheng H, Pinxian H, Guodong L, Xuebin C. Reliability and validity 549 evaluation of TCM syndrome scale for congestive heart failure. Journal of Traditional Chinese 550 Medicine. 2015;56(7):594-7. 
551 39. WANG Y, REN Y, Xuebin C, Xianghe W, Gang Z, Yuanhui H, et al. Evaluation of TCM 552 Syndrome Scales in Clinical Data of the Patients with Congestive Heart-failure. Gansu Journal of 553 Traditional Chinese Medicine. 2015;28(12):68-71.

554 40. Zhang P, Fu L, Jinjin L, Liu J, Liu Y, Zhu C, et al. Formulation and Evaluation of Clinical 555 Cross-sectional Information Collection Form in Chronic Cardiac Failure. Journal of Traditional 556 Chinese Medicine. 2011;52(4):295-8.

557 41. Association ECotNHaFPCoRUoMCP. Guidelines for the rational use of drugs for heart failure 558 (2nd edition). Chinese Journal of the Frontiers of Medical Science(Electronic Version). $5592019 ; 11(7): 1-78$.

560 42. Disease CBoCMAEBoCJoC. Chinese Journal of Cardiology 2015;42(2):98-122.

561 43. Christiansen TB and Lauritsen JM. (Ed.) EpiData - Comprehensive Data Management and 562 Basic Statistical Analysis System. Odense Denmark, EpiData Association, 2010-.

563 Http://www.epidata.dk

564 44. McMurray JJV, Packer M, Desai AS, Gong J, Lefkowitz MP, Rizkala AR, et al. Dual 565 angiotensin receptor and neprilysin inhibition as an alternative to angiotensin-converting enzyme 566 inhibition in patients with chronic systolic heart failure: rationale for and design of the Prospective 567 comparison of ARNI with ACEI to Determine Impact on Global Mortality and morbidity in Heart 568 Failure trial (PARADIGM-HF). EUR J HEART FAIL. 2013;15(9):1062-73. 